This is the post print version of the article, which has been published in Journal of Clinical Nursing. 2018, 27(3-4), e551-e558. http://dx.doi.org/10.1111/jocn.14093

MS. RIIKKA IKONEN (Orcid ID : 0000-0003-1526-3463)

Article type : Original Article

\title{
Preterm infants' mothers' initiation and frequency of breast milk expression and exclusive use of mother's breast milk in neonatal intensive care units
}

Concise title: Milk expression and breast milk usage in NICU

Authors

Riikka Ikonen, MNSc, doctoral candidate (Corresponding author)

Faculty of Social Sciences, University of Tampere

Institutional Address: PL 100, FI-33014 University of Tampere, Finland

E-mail: riikka.e.ikonen@uta.fi; tel: +358 505450402

Eija Paavilainen, $\mathrm{PhD}$, professor

Faculty of Social Sciences, University of Tampere

Etelä-Pohjanmaa Hospital District

Institutional Address: PL 100, FI-33014 University of Tampere, Finland

This article has been accepted for publication and undergone full peer review but has not been through the copyediting, typesetting, pagination and proofreading process, which may lead to differences between this version and the Version of Record. Please cite this article as doi: 10.1111/jocn.14093 
E-mail: eija.paavilainen@uta.fi; tel: +358 401904079

Mika Helminen, M.Sc., biostatistician

Science Center, Pirkanmaa Hospital District

Faculty of Social Sciences, University of Tampere

Mailing address: PL 100, FI-33014 University of Tampere, Finland

E-mail: mika.helminen@uta.fi; tel: +358 401901599

Marja Kaunonen, $\mathrm{PhD}$, professor

Faculty of Social Sciences, University of Tampere

General Administration, Pirkanmaa Hospital District

Institutional Address: PL 100, FI-33014 University of Tampere, Finland

E-mail: marja.kaunonen@uta.fi; tel: +358 401901454

Acknowledgements: The authors are grateful to head nurse Mika Vänskä, MHC, head nurse Maria Sukanen, MSc, and chief nursing officer Tarja Heino-Tolonen, MNSc, for their valuable collaboration in data collection. This study is funded by Doctoral Programme of Health Sciences, University of Tampere.

Contributions: The authors confirm that all authors meet the ICMJE criteria for authorship credit, as follows: (1) Substantial contributions to conception and design of, or acquisition of data or analysis and interpretation of data, (2) drafting the article or revising it critically for important intellectual content and (3) final approval of the version to be published.

Conflict of Interest: The authors declare that they have no conflict of interest. 


\section{ABSTRACT}

Aim and objectives. To describe preterm infants' mothers' expressing practices and exclusive use of mother's breast milk in neonatal intensive care settings, as well as to explore whether mothers' and infants' characteristics are predictors of the mother's inadequate expressing practices and non-exclusive use of mothers' breast milk.

Background. Use of their own mother's milk decreases preterm infants' mortality and morbidity, but expression is exhausting for the mothers. Mothers' and infants' characteristics are associated with milk output and exclusive breastfeeding at discharge, as well as later in infancy.

Design. Cross-sectional study.

Methods. The data were collected through questionnaires in two neonatal units. Multiple logistic regression was used to determine whether mothers' and infants' characteristics are predictors of late expression initiation ( $>$ six hours from birth), inadequate expression frequency ( $\leq$ six times per a day), and non-exclusive use of own mother's milk.

Results. The sample consisted of 129 mothers. One-third of the mothers had adequate expression practices. Half of the infants exclusively received their mother's own breast milk. Previous neonatal intensive care unit experience, poor psychological well-being, an infant's male gender, caesarean section, and high gestational birth age were significant predictors of late expression initiation. None of the studied variables were significant predictors of inadequate expression frequency. Furthermore, lack of previous expression experience, financial woes, and high gestational age were predictors of non-exclusive use of own mother's milk. 
Conclusions. Expression practices, as well as use of own mother's milk, were suboptimal. High gestational age was associated with both late expression initiation and non-exclusive breast milk use. The mothers maintained expression regardless of their well-being.

Relevance to clinical practice. Counseling and support is needed in order to avoid suboptimal expression practices. Special attention should be paid to mothers with moderately preterm infants, caesarean delivery, poor psychological well-being, and financial woes.

\section{SUMMARY BOX}

\section{What does this paper contribute to the wider global clinical community?}

- The associations between mothers' and infants' characteristics and breastfeeding are described in previous studies. This study's findings suggest that, in order to avoid delays in expression initiation, special attention should be paid to mothers with moderately preterm infants and male infants, as well as mothers with a caesarean section, poor psychological well-being, and previous neonatal intensive care unit (NICU) experience.

- Despite the well-established exhausting nature of expression, this study indicates that mothers maintain expression frequency despite their well-being or expression-related stress.

- Parallel to previous studies, mothers' previous expression experience was associated with exclusive use of their own breast milk in the NICU. Although the NICU environment can diminish the differences between socioeconomic classes, the family's financial woes predicted the non-exclusive use of the infants' own mothers' milk. 
Keywords. Preterm infants, Breastfeeding, Breast milk expression, Neonatal intensive care units

\section{INTRODUCTION}

In high income countries, $8.6 \%$ of infants are prematurely born before 37 completed gestational weeks (Blencowe et al. 2013). Despite the highly developed medical care in such countries, preterm birth is a leading cause of child mortality (Blencowe et al. 2013). The survival rates have increased during the last decades, and along with active risk pregnancy management, ventilation therapy, surfactant use, and infection prevention, the use of human milk has been detected as a reason for this advancement (Stoll et al. 2015). Exclusive use of human milk is highly recommended for preterm infants due its advantages to the infant's health and development (Edmond \& Bahl 2006). The lack of breast milk increases the rates of sepsis and necrotizing enterocolitis (NEC), which are both life-threatening conditions (American Academy of Pediatrics 2012). Furthermore, breast milk absence increases the risk of retinopathy of prematurity, in addition to cognitive problems later in childhood (American Academy of Pediatrics 2012). Exclusive use of the infant's own mother's breast milk (OMM) is particularly important in the neonatal intensive care unit (NICU), as pasteurized donor milk lacks important bioactive anti-infective components, such as secretory immunoglobulin A and lactoferrin (Heiman \& Schanler 2006), which play essential roles in controlling the gut's early colonization and protecting against NEC (Kim 2014). In addition, inadequate milk supply in the early lactation phase is associated with the use of artificial milk at week 12 postpartum (Hill et al. 2007), exposing the infant to artificial milk feeding risks, such as increased risks of common childhood infections, allergic diseases, diabetes, leukemia, and lymphoma (American Academy of Pediatrics 2012) later in childhood. 
Despite breast milk's advantages, breastfeeding rates are lower among preterm infants than term infants in Finland (National Institute for Health and Welfare 2012) and in Europe (Bonet et al. 2011). Lactation initiation and maintenance is more complicated for preterm infants' mothers. Milk production (lactogenesis II) has to be initiated by expression, because atbreastfeeding is not possible due to infants' immaturity and medical condition (Nyqvist et al. 2013). The mothers should initiate expression within six hours of delivery and express frequently (Nyqvist et al. 2013). Time of expressing initiation (Hill et al. 2005, Parker et al. 2012, Maastrup et al. 2014), as well as early frequency of stimulation (Hill et al 1999, Hill et al. 2001, Hill et al. 2005), have both been associated with milk output, adequate milk amount, and exclusive breastfeeding.

\section{BACKGROUND}

Expressing is an unexpected and new thing for mothers (Swanson et al. 2012). The mothers have to reevaluate their breastfeeding goals (Lee et al. 2009, Rossman et al. 2013), as well as learn several specific and unfamiliar practical skills (Swanson et al. 2012). Expressing supports the sense of motherhood in stressful situation, is important in establishing the connection between the mother and her infant, and is an important way to contribute to the NICU care (Lee et al. 2009, Swanson et al. 2012, Ikonen et al. 2016), but the strong link between mothering and expression also leads to perceiving expression as an obligatory task (Ikonen et al. 2016). Exhaustion is a common barrier to expression (Lee et al. 2009, Swanson et al. 2012, Hurst et al. 2013, Rossman et al. 2013). Furthermore, the transitions between home and the NICU impedes staying in expression schedules (Sisk et al. 2010), and mothers' compromised physical condition is another obstacle (Ikonen et al. 2016). Previous NICU experience and the presence of a family partner are detected as helpful factors for expression (Lee et al. 2009, Sisk et al. 2010, Ikonen et al. 2016). 
Some studies have examined factors associated with milk output and breastfeeding in preterm infants' mothers. The mothers' age (Hill et al. 2007), education level (Hill et al. 1999, Hill et al. 2005), annual income (Hill et al. 1999, Hill \& Aldag 2005, Hill et al. 2005), and previous breastfeeding experience (Hill et al. 1999, Maastrup et al. 2014) have all been found to associate with increased milk output and exclusive breastfeeding. However, these results are controversial, as other studies have not found such associations between breastfeeding and the mothers' education (Hill \& Aldag 2005, Hill et al. 2007, Maastrup et al. 2014), income (Hill et al. 2007), and previous breastfeeding experience (Hill et al. 2005, Hill \& Aldag 2005, Hill et al. 2007). Marital status and the mothers' mood states have not been found to be associated with milk output (Hill \& Aldag 2005, Hill et al. 2006, Hill et al. 2007), although maternal psychological stress and anxiety have been found to be risk factors for breastfeeding failure (Zanardo et al. 2011). The infant's gestational birth age is also associated with milk output (Hill et al. 2005) and breastfeeding at discharge (Maastrup et al. 2014), but again, the results are not uncontested (Hill et al. 1999, Hill et al. 2007). The infants' gender (Maastrup et al. 2014), as well as multiple births (Hill et al. 2007, Maastrup et al. 2014), are associated with breastfeeding at discharge and 12 weeks postpartum, whereas delivery type is not (Hill et al. 2007, Maastrup et al. 2014). Although there is a well-established association between adequate expression practices and adequate milk supply, the associations between socioeconomic factors and breastfeeding are not harmonious among preterm infants' mothers. Previous studies have described breastfeeding at discharge and later in infancy, but there is an information gap about factors related to exclusive use of OMM in NICU settings. Furthermore, the association of mother's and infant's characteristics with milk expression practices have not been investigated.

To conclude, the importance of early initiation and frequent expressing to adequate milk production has been demonstrated (Hill et al. 1999, Hill et al. 2001, Hill et al. 2005, Parker et 
al. 2012, Maastrup et al. 2014), as well as sociodemographic factors' relationship to milk production and breastfeeding (Hill et al. 1999, Hill et al. 2005, Hill \& Aldag 2005, Hill et al. 2006, Hill et al. 2007, Maastrup et al. 2014). However, the predictors for milk expressing practices and the exclusive use of OMM in NICU settings have not been described. Therefore, this study's objective was to describe preterm infants' mothers' expressing practices and exclusive breast milk use in NICU settings. Furthermore, the objective was to explore whether mothers' and infants' characteristics increase the odds for inadequate expressing practices and partial use of OMM in the NICU.

\section{METHODS}

\section{Study design}

This is a cross-sectional quantitative study.

\section{Data collection}

The sample size was determined with power analysis. For two-tailed independent samples $t$ test, $n=128$ is needed to achieve a power of $0 \cdot 80$ (Cohen's $d=0 \cdot 50, p=0 \cdot 05$ ) (Munro 2005). For multiple logistic regression, established guidelines for an adequate sample size do not exist (Munro 2005). It has been suggested that 10 events per covariate is a sufficient sample size, and in general, acceptable Confidence Interval (CI) coverage can be achieved using 5-9 events per covariate (Hosmer et al. 2013). In this sample, 45 mothers had the outcome event. This sample allows to add 4.5 variables to the model to avoid overestimation (Hosmer et al. 2013).

The data were collected in two NICUs between June 2015 and September 2016. One NICU provides intensive ( 12 beds) and intermediate care ( 17 beds) for sick and preterm infants without limits on gestation weeks. This NICU provides intensive-level care for its special 
responsibility area, which covers three special care nurseries in Southern Finland. The distance between the patient's home and the hospital can be nearly 300 kilometers. Another participating unit is a level II special care nursery with 10 beds. It provides care for moderately sick and preterm infants born later than 30 gestation weeks. Furthermore, very preterm infants are admitted to the unit after the intensive care phase. Both of the NICUs were open wards, only allowing the parents' presence during the daytime. Donor milk was available in both NICUs in case of insufficient milk supply, and the use of artificial milk was avoided during the hospitalization.

The inclusion criteria were as follows: a Finnish-speaking mother ( $\geq 18$ years) with singleton or twin preterm infant(s) (gestation age less than 37 weeks at birth or birth weight less than 2500 grams) admitted to the NICU. The infant(s) were more than six days old at the time of data collection, so daily expressing and lactogenesis II had been initiated. The presence of major crisis (related or nonrelated to the infant's medical status) were used as exclusion criteria; however, the mother could be eligible to participate later.

The data were collected through questionnaires. The primary investigator visited in the NICUs twice a week and identified the eligible mothers with a nurse's assistance. The questionnaire was administered to a nurse who was taking care of the family. The nurse recruited the mothers after receiving personal instructions from the primary investigator. This strategy was selected because the hospital administration and institutional review board did not recommended direct contact between a mother and the researcher. However, the investigator was available for further questions during the visits and was reachable by e-mail and phone. The mothers returned the filled consent forms and questionnaires in closed envelopes to the NICU nurses' stations, from where the researcher reclaimed them. 
The potential predictors of inadequate expression practices and non-exclusive OMM use in the NICU were selected based on previous findings. Based on the previous quantitative findings, it was suggested that mothers' age, education, financial situation, and previous NICU or expressing experience may predict inadequate expression practices. The rationale for this hypothesis is that these sociodemographic factors are associated with milk output and breastfeeding (Hill et al. 1999, Hill \& Aldag 2005, Hill et al. 2005, Hill et al. 2006, Hill et al. 2007, Maastrup et al. 2014), which, in turn, are strongly associated with adequate expressing practices. Furthermore, based on qualitative findings, the absence of a family partner, transitions between home and hospital, as well as compromised physical and psychological well-being and expression related stress are major burdens for expressing mothers (Lee et al. 2009, Sisk et al. 2010, Swanson et al. 2012, Hurst et al. 2013, Rossman et al. 2013, Ikonen et al. 2016). Therefore it was reasonable to hypothesize that these factors may interfere with optimal expression practices and predict non-excusive OMM use. The mothers' sociodemographic factors included age, marital status, education, the one-way distance from home to hospital (kilometers), previous NICU experience, and previous expression experience. The family's financial situation was measured with a self-rated 5-point semantic differential scale from $1=$ very poor to $5=$ very good. This approach was selected because we wanted to measure the family's perceived financial woes, rather than the family's socioeconomic class. The mother's physical and psychological well-being were also measured with single self-rated questions using a semantic differential scale from $1=$ very poor to $5=$ very good. Expression-related stress was measured with a visual analogue scale from $0=$ extremely stressful to $10=$ not at all stressful. Single question was selected because no scales measuring expression-related stress were available.

The infants' demographical factors were also selected based on quantitative and qualitative findings. Infants' gender, gestation birth age, and multiple births have been associated with 
milk output or breastfeeding (Hill et al. 2005, Hill et al. 2007, Maastrup et al. 2014). Furthermore, despite the previous evidence (Hill et al. 2007, Maastrup et al. 2014) delivery type was included as a potential predictor since recovery from major surgical operation may harden expression through different postpartum care practices and compromised physical health. Infants' age at the time of participation have been identified as a potential predictor since maintaining expressing frequency and adequate milk supply may be challenging due its very exhausting nature (Lee et al. 2009, Swanson et al. 2012, Hurst et al. 2013, Rossman et al. 2013). Therefore infants' demographical factors included gender, gestation birth age, delivery type, singleton / twin gestation, and age at the time of participation. Table 1 presents a dichotomization of the independent variables.

The outcomes were initiation (hours from delivery) and frequency (times per a day) of expressing. Inadequate expression practices were determined to be expressing initiation $>$ six hours after delivery and $\leq$ six times per day, based on previous literature (Hill et al. 1999, Hill et al. 2001, Nyqvist et al. 2013). Furthermore, exclusive use of breast milk was measured with the Index of Breastfeeding Status (IBS) (Labbok \& Krasovec 1990). The IBS scale measures the amount of the mother's own breast milk in relation to the total amount of the infant's enteral nutrition. The scale consists of seven points: 1) exclusive breastfeeding, no other milk; 2) high, over $80 \%$ of OMM; 3) medium high, 50-80\% of OMM; 4) medium low, $20-50 \%$ of OMM; 5) low, less than 20\% of OMM; 6) minimal or occasional use of OMM; and 7) none, no use of OMM at all. The mothers were asked to evaluate the current amount of their own breast milk and the total amount of milk needed daily. Vitamins, minerals, medications, human milk fortifiers, and parenteral nutrition were excluded. The first class (no other milk) was defined as those with exclusive use of OMM. 


\section{Statistical analysis}

The data were manually typed into SPSS $23 \cdot 0$ and checked for accuracy. Descriptive quantitative methods were used for frequencies, mean, and median values for central tendency measures, and standard deviation and percentiles for variation measures. Means and standard deviations were used for normally distributed variables, while medians and percentiles were reported if a variable was skewed (Munro 2005). Normal distribution was examined using Fisher's measure of skewness and Kolmogorov-Smirnov, while variables were determined to be skewed if $z<-1.96$ or $>1.96$, with $p<0.05$ (Munro 2005). Multiple logistic regression analysis (Munro 2005, Harrell 2001, Hosmer et al. 2013) was performed in order to estimate odds ratios (OR) and $95.0 \% \mathrm{CI}$ for inadequate expressing initiation and frequency, as well as the risk of non-exclusive OMM use (dependent variables). The independent variables were the mother's age (ratio), intimate relationship (yes/no), college or university level education (yes/no), hospital-home distance (ratio), previous NICU experience (yes/no), previous expression experience (yes/no), presence of financial woes (yes/no), good physical well-being (yes/no), good psychological well-being (yes/no), expression-related stress (ratio), infant's female gender (yes/no), gestational age at birth (ratio), vaginal delivery (yes/no), singleton infant (yes/no), and infant's age at the time of participation (ratio). The data consisted of six pairs of twins with different genders, with infant A's gender being used among these. The forward stepwise (likelihood ratio) method was used in order to achieve the data's most suitable model, in which several covariates are expected to associate together. This strategy was selected because it allows for building models sequentially, for eliminating redundant covariates, and for examining collections of models, rather than basing on binary covariate selection (Hosmer et al. 2013). As described earlier, the number of initial independent variables $(n=15)$ was large compared to the number of events $(n=45)$. However, because of strong support from previous qualitative and quantitative findings and 
recommendations to avoid binary covariate selection (Harrell 2001), all variables were included to the analysis. After adding significant variables in forward stepwise regression, the results of the analysis were checked to identify potential errors in models due to overestimation. In these analyses, multiple logistic regression with backward stepwise method was used. Backward stepwise elimination of non-significant variables resulted in consistent models with forward stepwise regression (data not shown), which supports the adequacy of the models.

The association of expression initiation to expression frequency and breastfeeding status, as well as expression frequency's association to breastfeeding status, were studied with MannWhitney $U$-test (Munro 2005). A statistical significance level of $5 \cdot 0 \%$ was used in all analyses.

\section{Ethical aspects}

The Declaration of Helsinki ethical guidelines (World Medical Association 1964) have been followed. The study was approved by the Regional Ethics Committee of the Expert Responsibility area of Tampere University Hospital (R15100H). Written information was provided for all potential participants, and the opportunity to ask for additional information was offered prior to their decision to participate. Written consent was obtained from the participants. The participants' anonymity and confidentiality were protected. The mothers had daily contact with health care via their infants in NICU, and therefore, their psychological well-being was routinely monitored after the participation. 


\section{RESULTS}

\section{Demographic characteristics}

In total, 133 questionnaires were returned, with a response rate of $61 \cdot 6 \%$. Participants were further excluded $(n=4)$ if the mother's infant was less than six days old $(n=2)$, or if a substantial amount $(>50 \%)$ of data were missing in the questionnaire $(n=2)$. The mothers $(n=129)$ were, on average, $31 \cdot 3$ years old $(\mathrm{SD}=5 \cdot 2)$ and mainly primiparous $(54 \cdot 3 \%, n=$ 70). The infants were born at 32.6 gestational weeks $(\mathrm{SD}=3 \cdot 1)$, and they were 1.8 weeks old $(\mathrm{SD}=1 \cdot 1)$ at the time of data collection. The data consisted of 27 pairs $(20 \cdot 9 \%)$ of twins.

Demographic characteristics are presented in Table 1.

\section{Expressing initiation, expressing frequency and breast milk use}

The median expression initiation time was $9 \cdot 0$ hours $\left(\mathrm{Q}_{1}-\mathrm{Q}_{3}=5 \cdot 0-24 \cdot 0\right)$, while median expression frequency was $6 \cdot 0$ times per a day $\left(\mathrm{Q}_{1}-\mathrm{Q}_{3}=5 \cdot 0-7 \cdot 0\right)$. Overall, 36.0\% $(n=45)$ of mothers initiated expression with six hours from birth, and 33.3\% $(n=43)$ of mothers expressed more than six times per day. Half of the mothers $(49 \cdot 2 \%, n=63)$ offered their own breast milk exclusively.

\section{Predictors for inadequate expression practices and non-exclusive breast milk use}

Independent significant predictors for late expression initiation (i.e., $>6$ hours from birth) were the mother's previous NICU experience, the infant's male gender, the mother's poor psychological well-being, caesarean section, and the length of gestation at the time of birth $(p$ $<0 \cdot 05$, Table 2$)$. None of the predictors were significantly associated with adequate expression frequency. Furthermore, three covariates were independently associated with nonexclusive OMM use: lack of previous expressing experience, poor economic status, and higher infant's gestation birth age $(p<0 \cdot 05$, Table 3$)$. 


\section{Associations between expression practices and breast milk use}

Mothers who initiated expression within 6 hours of birth offered a significantly higher amount of breast milk for their infants than mothers who initiated expression later $(\mathrm{Md}=1 \cdot 0$, $\mathrm{Q}_{1}-\mathrm{Q}_{3}=1 \cdot 0-2 \cdot 0$ vs. $\mathrm{Md}=2 \cdot 0, \mathrm{Q}_{1}-\mathrm{Q}_{3}=1 \cdot 0-3 \cdot 0, p=0 \cdot 020$, respectively). Adequate and late expression initiation was not found to be associated with expression frequency $\left(\mathrm{Md}=6 \cdot 0 \mathrm{Q}_{1^{-}}\right.$ $\mathrm{Q}_{3}=5 \cdot 0-7 \cdot 0$ vs. $\mathrm{Md}=6 \cdot 0 \mathrm{Q}_{1}-\mathrm{Q}_{3}=5 \cdot 0-7 \cdot 0, p=0 \cdot 122$, respectively). Among the mothers who expressed six or more times per day, the milk amount was greater than mothers who expressed less frequently, but the difference was non-significant $\left(\mathrm{Md}=1 \cdot 0, \mathrm{Q}_{1}-\mathrm{Q}_{3}=1 \cdot 0-3 \cdot 0\right.$ vs. $\mathrm{Md}=2 \cdot 0 \mathrm{Q}_{1}-\mathrm{Q}_{3}=1 \cdot 0-3 \cdot 0, p=0 \cdot 161$, respectively).

\section{DISCUSSION}

This study was conducted in order to describe preterm infants' mothers' expressing practices and exclusive use of breast milk, as well as to explore whether socioeconomic factors, the family's life burdens, and the infants' characteristics are risk factors for inadequate expressing practices and non-exclusive breast milk use in NICU settings. The study reveals that the expression initiation time, expression frequency, and mother's breast milk use were suboptimal. To the authors' knowledge, this is the first study that has examined the predictors for inadequate expression practices and NICU breast milk use.

Associations between expression initiation, frequency, and milk output have been established in previous studies. Initiation of expression is associated with milk output and breastfeeding (Hill et al. 2005, Parker et al. 2012, Maastrup et al. 2014). Similarly, in this study, early expression initiation was associated with exclusive OMM use. In this study, only one-third of the mothers expressed more than six times per day, which is indicated to be the minimal frequency for adequate milk supply (Hill et al. 1999, Hill et al. 2001, Hill et al. 2005, Nyqvist et al. 2013). The expression frequency, however, was not significantly associated 
with exclusive OMM use. The most apparent reason for this is that the participation time was less than two weeks postpartum. The milk amount is hormonally controlled during this phase, and autocrine control transfer (demand based) occurs at six weeks after delivery (Riordan 2010). However, early expression frequency is associated with milk output and adequate milk amount at two to six weeks (Hill et al. 2001, Hill et al. 2005). In addition, half of the mothers had an inadequate milk supply to fulfill their infant's requirements in the early lactation phase. This is identified as a risk factor for inadequate milk output later (Hill et al. 2005).

Infants' high gestational birth age increased the risk for late expression initiation and nonexclusive breast milk use. This finding is contrary to previous studies' findings, which have shown that preterm infant's low gestational age is associated with milk output (Hill et al. 2005) and exclusive breastfeeding (Maastrup et al. 2014), although some studies did not find such association (Hill et al. 1999, Hill et al. 2007). One explanation for this finding is that mothers of preterm infants are counselled to initiate expression immediately after delivery due to trophic feeding advantages (i.e., administration of very small OMM doses from day one) (Edmond \& Bahl 2006). Contrarily, such importance might not have been seen among moderately preterm infants, which are not generally in critical condition. However, this study's findings indicate that moderately preterm infants had greater risk of non-exclusive OMM use, emphasizing the importance of early expression initiation in moderately preterm infants' mothers.

Infant's male gender was a strong and significant predictor for late expression initiation. Interestingly, this finding is supported by findings from other Nordic countries, as Maanstup et al. (2014) found that male gender is a risk factor for non-exclusive breastfeeding at discharge. Other previous studies (Hill et al. 2007, Bonet et al. 2011) have not investigated the association between infant's gender and breastfeeding. The results suggest that infant's 
gender could be associated with expression initiation and breastfeeding, but the underlying reasons are unknown.

Findings regarding the mother's previous experience were controversial. The mother's previous NICU experience was a risk factor for late expression initiation, which was contrary to previous qualitative findings indicating that previous experience is a helpful factor for the mothers (Ikonen et al. 2016). One possible explanation for this finding could be inadequate counseling and unjustified reliance on mother's previous experience by the health care staff. However, parallel to previous qualitative findings (Ikonen et al. 2016), previous expression experience was associated with exclusive OMM use. This finding is further supported by Hill et al (1999) and Maastrup et al. (2014), although opposite findings have also been reported (Hill \& Aldag 2005, Hill et al. 2005, Hill et al. 2007).

The birth of a preterm infant, NICU admission, and expression are stressful demands on the mothers (Lee et al. 2009, Swanson et al. 2012, Ikonen et al. 2016). In this study, the mother's poor psychological well-being was a risk for late initiation. However, the results indicate that mothers maintain their expression frequency despite their poor physical or psychological condition, or their expression-related stress. Despite the exhausting nature of expression (Lee et al. 2009, Swanson et al. 2012, Hurst et al. 2013, Rossman et al. 2013, Ikonen et al. 2016), expression is viewed as an obligation (Ikonen et al. 2016), and mothers decrease frequency and cease expression as the final coping strategy in an unmanageable situation (Lee et al. 2009, Swanson et al. 2012). Similarly, poor psychological condition or expression-related stress were not associated with exclusive OMM use. Neuroendocrine responses to stress and lactation are related (Hill et al. 2003), and psychological stress has been found to associate with breastfeeding failure (Zanardo et al. 2011). Despite these findings, the mother's mood states are not associated with milk output (Hill et al. 2006), supporting this study's findings. 
Socioeconomical differences were partly apparent in this study. The family's financial woes increased the risk of non-exclusive OMM use in NICU settings, which is also supported by previous studies (Hill et al. 1999, Hill \& Aldag 2005, Hill et al. 2005). However, the mother's education was not associated with exclusive OMM use, as supported by Maastrup et al. (2014). Notably, Hill et al. $(1999,2005,2007)$ have presented opposite findings in the United States. Although high maternal education is associated with breastfeeding in the Finnish population (National Institute for Health and Welfare 2012), the NICU environment and counseling could diminish this association.

This study has some limitations. All of the participants were Caucasian and relatively highly educated, which limits the results' transferability. The presence of a major crisis was used as exclusion criteria due to ethical reasons. These exclusion reasons were related to the infant's extremely critical condition, but in order to protect the patient's privacy, exact diagnoses were not collected from excluded mothers. However, for this reason, the results are not representative for mothers with extremely critically ill infants. Furthermore, donor milk availability in NICUs limits the results' transferability to NICU settings without such an advantage. Long maternity leaves and extensive welfare benefits in Finland further limits the results' transferability to different societies. Some compromises had to be done in selecting covariates. Previous qualitative and quantitative findings indicated that a large amount of independent variables are potentially related to outcome variables. Therefore forward addition in logistic regression was applied in this relatively small sample, despite the presented recommendations (Harrell 2001, Hosmer et al. 2013). This limitation was partly diminished by testing the regression models using backward stepwise method. Physical and psychological well-being were measured with single self-rated questions rather than validated scales, which is a threat to validity. 


\section{CONCLUSION}

The study indicates that only one of the three mothers initiated expression within the recommended time and maintained adequate expression frequency. Furthermore, nonexclusive OMM use was common. Mothers with a moderately preterm infant, male infant, poor psychological condition, previous NICU experience, and a caesarean section have increased risk of inadequate expression initiation. Furthermore, the infant's high gestational age was also a predictor for non-exclusive OMM use. The results suggest that inadequate expression frequency is not explained by the mother's or the infant's characteristics, or by the mother's well-being and stress. Socioeconomic differences are partially apparent in the NICU, as financial woes predicted non-exclusive OMM use. Furthermore, previous expression experience was a predictor for exclusive OMM use. Further studies are needed for detecting the controversial associations between the mothers' previous experiences and expression initiation, as well as their exclusive OMM use. Furthermore, this study found an interesting association between male gender infants and suboptimal expression initiation, which should be studied further.

\section{RELEVANCE TO CLINICAL PRACTICE}

The preterm infants' mothers' expression practices, as well as exclusive OMM use in NICU settings, were suboptimal in this study. Counseling and support is needed (Nyqvist et al. 2013) in order to diminish the differences in breastfeeding rates among term and preterm infants (Bonet et al. 2011, National Institute for Health and Welfare 2012). More specifically, the nurses should encourage the mothers to initiate expression within six hours of birth, as well as to express frequently (Nyqvist et al. 2013). According to this study's results, special attention should be paid to mothers with previous NICU experience and compromised psychological well-being, as well as to mothers whose infant is born via caesarean section, is 
moderately preterm, and is a boy. Although qualitative studies have indicated that the mother's exhaustion and stress are expression barriers (Lee et al. 2009, Swanson et al. 2012, Hurst et al. 2013, Rossman et al. 2013), this study's results suggest that mothers maintain expression regardless of their physical or psychological well-being, or their expressionrelated stress. Nevertheless, the mother's emotional well-being should be routinely monitored and supported by the NICU staff. In addition, close collaboration with social work is needed, as socioeconomic differences in breastfeeding are also apparent in NICU settings.

Number of words used (excluding abstract, references, tables, and figures): 4609

\section{REFERENCES}

American Academy of Pediatrics (2012) Breastfeeding and the use of human milk. Pediatrics 129, e827-e841.

Blencowe H, Cousens S, Chou D, Oestergaard M, Say L, Moller AB, Kinney M \& Lawn J (2013) Born too soon: The global epidemiology of 15 million preterm births. Reproductive Health 10, S2.

Bonet M, Blondel B, Agostino R, Combier E, Maier RF, Cuttini M, Khoshnood B \& Zeitlin J (2011) Variations in breastfeeding rates for very preterm infants between regions and neonatal units in Europe: results from the MOSAIC cohort. Archives of Disease in Childhood Fetal and Neonatal Edition 96, F450-F452.

Edmond K \& Bahl R (2006) Optimal feeding of low-birth-weight infants: Technical review. World Health Organization, Geneva. Available at: 
http://www.who.int/maternal_child_adolescent/documents/9241595094/en/ (accessed 29 October 2016).

Harrell FE, Jr (2001) Regression modeling strategies. With applications to linear models, logistic regression, and survival analysis. Springer, New York.

Heiman H \& Schanler RJ (2006) Benefits of maternal and donor human milk for premature infants. Early Human Development 82, 781-787.

Hill PD, Aldag JC \& Chatterton RT (1999) Effects of pumping style on milk production in mothers of non-nursing preterm infants. Journal of Human Lactation 15, 209-216.

Hill PD, Aldag JC \& Chatterton RT (2001) Initiation and frequency of pumping and milk production in mothers of non-nursing preterm infants. Journal of Human Lactation 17, 9-13.

Hill PD, Chatterton RT, Aldag JC (2003) Neuroendocrine responses to stressors in lactating and nonlactating mammals: A literature review. Biological Research for Nursing 5, 79-86.

Hill PD \& Aldag JC (2005) Milk volume on day 4 and income predictive of lactation adequacy at 6 weeks of mothers of nonnursing preterm infants. Journal of Perinatal and Neonatal Nursing 19, 273-282.

Hill PD, Aldag JC, Chatterton RT \& Zinaman M (2005) Primary and secondary mediators' influence on milk output in lactating mothers of preterm and term infants. Journal of Human Lactation 21, 138-150.

Hill PD, Aldag JC, Demirtas H, Zinaman M \& Chatterton RT (2006) Mood states and milk output in lactating mothers of preterm and term infants. Journal of Human Lactation 22, 305314. 
Hill PD, Aldag JC, Zinaman M \& Chatterton RT (2007) Predictors of preterm infant feeding methods and perceived insufficient milk supply at week 12 postpartum. Journal of Human Lactation 23, 32-38.

Hosmer DW, Lemeshow S \& Sturdivant RX (2013) Applied logistic regression. 3rd edn. Wiley, New Jersey.

Hurst N, Engebretson J \& Mahoney JS (2013) Providing mother's own milk in the context of the NICU: a paradoxical experience. Journal of Human Lactation 29, 366-373.

Ikonen R, Paavilainen E \& Kaunonen M (2016) Trying to live with pumping: Expressing milk for preterm or small for gestation age infants. The American Journal of Maternal Child Nursing 41, 110-115.

Kim JH (2014) Necrotizing enterocolitis: The road to zero. Seminars in Fetal \& Neonatal Medicine 19, 39-44.

Labbok M \& Krasovec K (1990) Toward consistency in breastfeeding definitions. Studies in Family Planning 21, 226-230.

Lee TY, Lee TT \& Kuo S (2009) The experiences of mothers in breastfeeding their very low birth weight infants. Journal of Advanced Nursing 65, 2523-2531

Maastrup R, Hansen BM, Kronborg H, Bojesen SN, Hallum K, Frandsen A, Kyhnaeb A, Svarer I \& Hallström I (2014) Factors associated with exclusive breastfeeding of preterm infants. Results from a prospective national cohort study. PLOS One 9, e89077 Munro BH (2005) Statistical Methods for Health Care Research. 5th edn. Lippincott Williams \& Wilkins, Philadelphia. 
National Institute for Health and Welfare (2012) Infant feeding in Finland 2010. Report 8/2012. National Institute for Health and Welfare, Helsinki.

Nyqvist KH, Häggkvist AP, Hansen MN, Kylberg E, Frandsen AL, Maastrup R, Ezeonodo A, Hannula L \& Haiek LN (2013) Expansion of the Baby-Friendly Hospital Initiative Ten Steps to Successful Breastfeeding into neonatal intensive care: expert group recommendations. Journal of Human Lactation 29, 300-309

Parker LA, Sullivan S, Krueger C, Kelechi T \& Mueller M (2012) Effect of early breast milk expression on milk volume and timing of lactogenesis stage II among mothers of very low birth weight infants: a pilot study. Journal of Perinatology 32, 205-209.

Riordan J (2010) Anatomy and physiology of lactation. In Breastfeeding and Human Lactation (Riordan J \& Wambach K ed.), 4th edn. Jones and Bartlett Publishers, Massachusetts, pp. 79-111.

Rossman B, Kratovil AL, Greene MM, Engstrom JL \& Meier PP (2013) "I have faith in my milk": the meaning of milk for mothers of very low birth weight infants hospitalized in the neonatal intensive care unit. Journal of Human Lactation 29, 359-365

Sisk P, Quandt S, Parson N \& Tucker J (2010) Breast milk expression and maintenance in mothers of very low birth weight infants: supports and barriers. Journal of Human Lactation 26, $368-375$

Stoll BJ, Hansen NI, Bell EF, Walsh MC, Carlo WA, Shankaran S, Laptook AR, Sanchez PJ, Van Meurs KP, Wyckoff M, Das A, Hale EC, Ball MB, Newman NS, Schibler K, Poindexter BB, Kennedy KA, Cotten CM, Watterberg KL, D'Anglio CT, DeMauro SB, Truog WE, Devaskar U \& Higgins RD (2015) Trends in care practices, morbidity, and mortality of 
extremely preterm neonates 1993-2012. The Journal of the American Medical Association 314, 1039-1051.

Swanson V, Nicol H, McInnes R, Cheyne H, Mactier H \& Callander E (2012) Developing maternal self-efficacy for feeding preterm babies in the neonatal unit. Qualitative Health Research 22, 1369-1382

World Medical Association (1964) World Medical association declaration of Helsinki.

Ethical Principles for Medical Research Involving Human Subjects. Available at: http://www.wma.net/en/30publications/10policies/b3 (accessed 29 October 2016).

Zanardo V, Gambina I, Begley C, Litta P, Cosmi E, Giustardi A \& Trevisanuto D (2011) Psychological distress and early lactation performance in mothers of late preterm infants. Early Human Development 87, 321-323.

Table 1 Characteristics of participants $(n=129)$

\begin{tabular}{ll}
\hline Variable & Descriptive statistics
\end{tabular}

\section{Mothers}

Age, years, $M(\mathrm{SD})$

$31 \cdot 3(5 \cdot 2)$

Marital status, $n(\%)$

In relationship $119(92 \cdot 2)$

Single $10(7 \cdot 8)$

Completed degree, $n(\%)$ 
College or university

Vocational school or less

Hospital-home distance, kilometers, $M d\left(\mathrm{Q}_{1}-\mathrm{Q}_{3}\right) \quad 20 \cdot 0(8 \cdot 0-51 \cdot 5)$

Previous NICU experience, $n(\%)$

No

Yes

Previous expression experience, $n(\%)$

No

Yes

Financial woes $n(\%)$

No $(3 \cdot 1-5)$

Yes $(1-3 \cdot 0)$

Physical well-being, $n(\%)$

Good $(3 \cdot 1-5)$

Poor $(1-3 \cdot 0)$

Psychological well-being, $n(\%)$

Good $(3 \cdot 1-5)$

Poor $(1-3 \cdot 0)$
$114(88 \cdot 4)$

$72(55 \cdot 8)$

$57(44 \cdot 2)$

$15(11 \cdot 6)$

$75(58 \cdot 1)$

$54(41 \cdot 9)$

$73(56 \cdot 6)$

$56(43 \cdot 4)$

$80(62 \cdot 0)$

49 (38.0)

$79(61 \cdot 7)$

$49(38 \cdot 3)$ 
Infants

Pregnancy, $n(\%)$

Singleton

Twin

GA at birth, $M(\mathrm{SD})$

Delivery type, $n(\%)$

Vaginal

Caesarean

Gender, $n(\%)$

Female

Male

Age, weeks, $M(\mathrm{SD})$
$102(79 \cdot 1)$

$27(20 \cdot 9)$

$32 \cdot 6(3 \cdot 1)$

$65(50 \cdot 4)$

$64(49 \cdot 6)$

$58(45 \cdot 0)$

$71(55 \cdot 0)$

$1 \cdot 8(1 \cdot 1)$ 
Table 2 Predictors of late expression initiation (> six hours from delivery) - results of regression modeling $(n=125)$

\begin{tabular}{lllll}
\hline Variable & Reference category & OR $^{\text {adjusted }}$ & $95 \% \mathrm{CI}$ & $p$
\end{tabular}

\begin{tabular}{lllll}
\hline Previous NICU experience $^{\mathrm{a}}$ & Previous experience, no & 4.52 & $1 \cdot 01-20 \cdot 36$ & 0.049
\end{tabular}

Infant's gender

Female

$3 \cdot 29$

$1 \cdot 39-7 \cdot 82 \quad 0 \cdot 007$

Delivery type ${ }^{\mathrm{a}}$

Vaginal delivery

$3 \cdot 06$

$1 \cdot 28-7 \cdot 31 \quad 0 \cdot 012$

Psychological well-being ${ }^{\mathrm{a}}$

Good (score $>3.0$ of 5) $\quad 3.03$

$1 \cdot 21-7 \cdot 60 \quad 0 \cdot 018$

Gestational age at birth ${ }^{\mathrm{b}}$

For one more day

$1 \cdot 29$

$1 \cdot 11-1 \cdot 50 \quad 0 \cdot 001$

Variables not in equation after forward elimination: Mother's age ${ }^{\mathrm{b}}$, marital status ${ }^{\mathrm{a}}$, education $^{\mathrm{a}}$, hospital-home distance ${ }^{\mathrm{b}}$, previous expression experience ${ }^{\mathrm{a}}$, financial woes ${ }^{\mathrm{a}}$, physical well-being ${ }^{\mathrm{a}}$, expression-related stress $^{\mathrm{b}}$, multiple birth $^{\mathrm{a}}$, infant's age $^{\mathrm{b}}$

Goodness of fit (Hosmer and Lemeshow test) $p=0.778$

Nagelkerke $R^{2}=0 \cdot 246$

$\mathrm{OR}^{\text {adjusted }}$, adjusted odds ratio

95\% CI, 95\% confidence interval for adjusted odds ratio

${ }^{a}$ Binary variable

${ }^{\mathrm{b}}$ Ratio variable 
Table 3 Predictors of non-exclusive use of OMM - results of regression modeling $(n=127)$

\begin{tabular}{lllll}
\hline Variable & Reference category & OR $^{\text {adjusted }}$ & $95 \%$ CI & $p$ \\
& & & & \\
\hline Financial woes $^{\mathrm{a}}$ & No (score > 3.0 of 5) & 2.96 & $1.36-6.43$ & 0.006 \\
Previous expression experience $^{\mathrm{a}}$ & Previous experience, yes & 2.54 & $1.16-5.59$ & 0.020 \\
& & & & \\
Gestational age at birth $^{\mathrm{b}}$ & For one more day & 1.22 & $1.07-1.39$ & 0.002
\end{tabular}

Variables not in equation after forward elimination: Mother's age ${ }^{\mathrm{b}}$, marital status ${ }^{\mathrm{a}}$,

education $^{\mathrm{a}}$, hospital-home distance ${ }^{\mathrm{b}}$, previous NICU experience ${ }^{\mathrm{a}}$, physical well-being ${ }^{\mathrm{a}}$,

psychological well-being $^{\mathrm{a}}$, expression-related stress $^{\mathrm{b}}$, infant's gender $^{\mathrm{a}}$, delivery type $^{\mathrm{a}}$,

multiple birth ${ }^{\mathrm{a}}$, infant's age ${ }^{\mathrm{b}}$

Goodness of fit (Hosmer and Lemeshow test) $p=0 \cdot 852$

Nagelkerke $R^{2}=0 \cdot 203$

OMM, own mother's breast milk

$\mathrm{OR}^{\text {adjusted }}$, adjusted odds ratio

95\% CI, 95\% confidence interval for adjusted odds ratio

${ }^{a}$ Binary variable

${ }^{\mathrm{b}}$ Ratio variable 